\title{
Needle puncture injury of the rat intervertebral disc affects torsional and compressive biomechanics differently
}

\author{
Arthur J. Michalek · Kristin L. Funabashi • \\ James C. Iatridis
}

Received: 7 December 2009/Revised: 15 May 2010/Accepted: 23 May 2010/Published online: 11 June 2010

(c) Springer-Verlag 2010

\begin{abstract}
Needle puncture is a common method of inducing intervertebral disc (IVD) degeneration in small animal models and may have some similarities to IVD injury conditions such as herniation. Yet, the influence of puncture injuries on IVD biomechanics is not well understood. This study quantified the acute effects of anular injury on the biomechanics of rat caudal IVDs in compression and torsion following puncture with 30,25 and $21 \mathrm{G}$ needles. In compression, puncture injury reduced elastic stiffness by $20 \%$ for all needle sizes, but differences between control and punctured discs did not remain after compressive overload. In contrast, torsional parameters associated with anular fiber tension were affected proportionally with needle size. We conclude that IVD injuries that penetrate through the thickness of the annulus affect IVD biomechanics through different mechanisms for compression and torsion. Anular injuries affect torsional properties in a manner directly related to the amount of fiber disruption and compressive properties in a manner that affects pressurization.
\end{abstract}

Keywords Intervertebral disc $\cdot$ Needle puncture . Injury · Biomechanics

\footnotetext{
A. J. Michalek · K. L. Funabashi

College of Engineering and Mathematical Sciences,

University of Vermont, Burlington, VT, USA

J. C. Iatridis $(\square)$

Spine Bioengineering Laboratory, University of Vermont,

201 Perkins Building, 33 Colchester Ave,

Burlington, VT 05405, USA

e-mail: james.iatridis@uvm.edu
}

\section{Introduction}

Intervertebral discs (IVDs) provide connectivity between adjacent vertebrae in the spine while also enabling mobility. IVD degeneration is associated with mechanical damage and enzymatic degradation through accumulated micro-trauma episodes as well as from larger acute injuries. IVDs are also the largest avascular structures in the body making them very slow to repair [1]. A literature review concluded that acute mechanical injury of the annulus fibrosus (AF) may lead directly to degenerative changes, with biomechanical consequences depending on both the type of injury and mode of loading [2]. The foundation of this dependence is the classification of loading modes based on whether the AF is recruited primarily to constrain nucleus pulposus (NP) fluid pressure (compression, bending) [3] or to provide vertebral attachment (torsion, shear) [4]. To explore this phenomenon in the context of injury size, the present study focuses on compression and axial torsion. These loading modes are axisymmetric relative to injury location and are also the bounding cases for NP pressurization-dominated (compression) and AF fiber tension-dominated (torsion) load carriage. Successful anular repair depends on a clear understanding of how structural damage alters the ability of the AF to perform these two roles.

While human disc degeneration is a complex and multifactorial process that occurs over decades, it remains clear that focal anular injury is a potential initiator of disc degeneration [2]. For example, discography has recently been linked to accelerated degeneration in human lumbar discs [5]. IVDs subjected to needle puncture also show progressive degenerative changes in vivo, a decrease in IVD height [6-11] and a loss of compressive stiffness [8, 12] under in vitro testing conditions. The needle puncture 
model has gained acceptance for research purposes, because it is easy to implement with a high degree of repeatability particularly for in vivo models. To date, investigations into the effects of acute injury on disc biomechanics have focused primarily on large animal models [13-18]. There has been little attention paid to biomechanics immediately following acute injury of small animal IVDs [19]. In addition to showing certain biomechanical similarities with human IVDs [20-23], small animals offer convenient in vivo models for degenerative and regenerative procedures. The rat tail model, in particular, is an ideal choice for in vivo disc studies due to its high accessibility, relatively low cost and axisymmetry. For such models to provide effective platforms for in vivo testing of AF repair, an improved mechanical understanding of how small animal IVDs are altered by injury is required.

This study quantifies the influence of acute injury on the AF's ability to fulfill the dual roles of containing NP fluid pressure and providing vertebral connectivity. The primary hypothesis is that AF injury affects compressive and torsional mechanics through different mechanisms detectable via distinct patterns of elastic and viscoelastic alterations in response to increasing needle puncture size. The second hypothesis is that needle injury creates biomechanical damage, which predisposes rat caudal IVDs to successive damage. The hypotheses were tested by in vitro loading of rat caudal IVDs in compression and torsion before and after needle puncture injuries with three different needle gages. The effect of altered fluid pressurization on compressive mechanics was further tested by reduction in tissue water content induced by compressive overload, and the influence of anular fiber integrity on failure mechanics was tested by a torsional quasi-static ramp to failure. Needle sizes included the feasible experimental range, from the smallest gage that allowed insertion through the AF without substantial needle bending (30 G), a moderate needle size $(25 \mathrm{G})$ and a large needle size that roughly spanned the entire rat caudal IVD height $(21 \mathrm{G})$.

\section{Methods}

\section{Specimen preparation}

Three motion segments consisting of two vertebrae and an IVD, levels c6-7 through c10-11, were obtained from the tails of 43 rats. Following the removal of skin and other surrounding tissue, the vertebrae were potted in stainless steel tubes using cyanoacrylate. Removal of tail musculature allowed isolation of the disc's contribution to compressive and torsional mechanics. A custom-built potting jig ensured that the pots were parallel and concentric with motion segments. For compressive and torsional tests, potted motion segments were held in stainless steel grips and immersed in a bath of normal PBS. Discs were systematically assigned to one of four groups (unpunctured control and injury from 30, 25 and $21 \mathrm{G}$ needles).

Previous studies described difficulties in consistently puncturing small IVDs with large needles [12]. A puncture injury that fully penetrates the rat $\mathrm{AF}$ with a large syringe needle (with standard long bevel) will also damage the opposite side of the AF. To ensure consistent injuries across all three needle sizes, punctures were carried out using syringe needles modified to emulate micro-biopsy punches (Fig. 1a) with cutting edges along the inside surface. Based on an average IVD diameter of $4 \mathrm{~mm}$, the needles were marked with tape at a depth of $2 \mathrm{~mm}$ to ensure penetrating through the AF at a consistent depth. Micrographs of transversely cryosectioned discs (Fig. 1b) show AF material removed to an extent increasing with needle size. Histologically, the injuries appear to be slightly smaller than the nominal inside diameters of the needles $(30 \mathrm{G}=$ $0.140 \mathrm{~mm}, 25 \mathrm{G}=0.241 \mathrm{~mm}$ and $21 \mathrm{G}=0.495 \mathrm{~mm}$ ), suggesting some relaxation in the tissue following puncture. Extrusion of NP material was not observed upon puncture, and NP material was still present in transverse sections with little movement into the needle track.

\section{Compressive testing}

Compressive biomechanics were evaluated using an axial testing machine (ELF 3200, Bose Corp) using a displacement controlled test protocol (Fig. 2a). IVDs were first compressed by $0.35 \mathrm{~mm}$, [24] previously determined to be average equilibrium displacement under resting stress of $0.15 \mathrm{MPa}$ [25], and allowed to relax for $30 \mathrm{~min}$ (Pre). The compressive pre-loading step accounted for axial swelling of the disc, which followed removal of surrounding tissue. Compressive stress did not exceed $0.24 \mathrm{MPa}$ during any pre-load. Motion segments underwent a cyclic compressive test (T1) consisting of five cycles of displacement between 0 and $-0.45 \mathrm{~mm}$ at both 0.05 and $0.1 \mathrm{~Hz}$, the final cycle of which was used for analysis. Motion segments were then returned to resting height for $5 \mathrm{~min}$ (DW), during which the punctured specimens were punctured and the control specimens were not (control: $n=21,30 \mathrm{G}: n=21,25 \mathrm{G}$ : $n=21,21 \mathrm{G}: n=20$ ), followed by a second dynamic compressive test (T2). Disc hydration was reduced by a hyperphysiological axial compressive overload, consisting of a ramp to $80 \%$ of resting height at a rate of $0.1 \% / \mathrm{s}$ followed by an immediate return to resting height (OL). This height loss was estimated to result in an $8 \%$ reduction in NP water content [24]. A third dynamic compressive test was performed (T3) after $100 \mathrm{~s}$. Compressive biomechanical data were analyzed using a custom written Matlab code, which determined the storage $\left(K^{\prime}\right)$ and loss $\left(K^{\prime \prime}\right)$ 
Fig. 1 Left original and modified hypodermic needles. Needles were modified to the shape of a biopsy so that AF penetrating needle injury in the small rat IVD ( $\sim 2 \mathrm{~mm}$ radius) was constrained to a single hole that did not puncture both sides of the AF. Right representative polarized light micrographs of transversely cryosectioned discs of control (a), and specimens with needle injury with $30 \mathrm{G}$ (b), $25 \mathrm{G}$ (c) and $21 \mathrm{G}(\mathbf{d})$. For needle injury groups, arrows indicate injury location

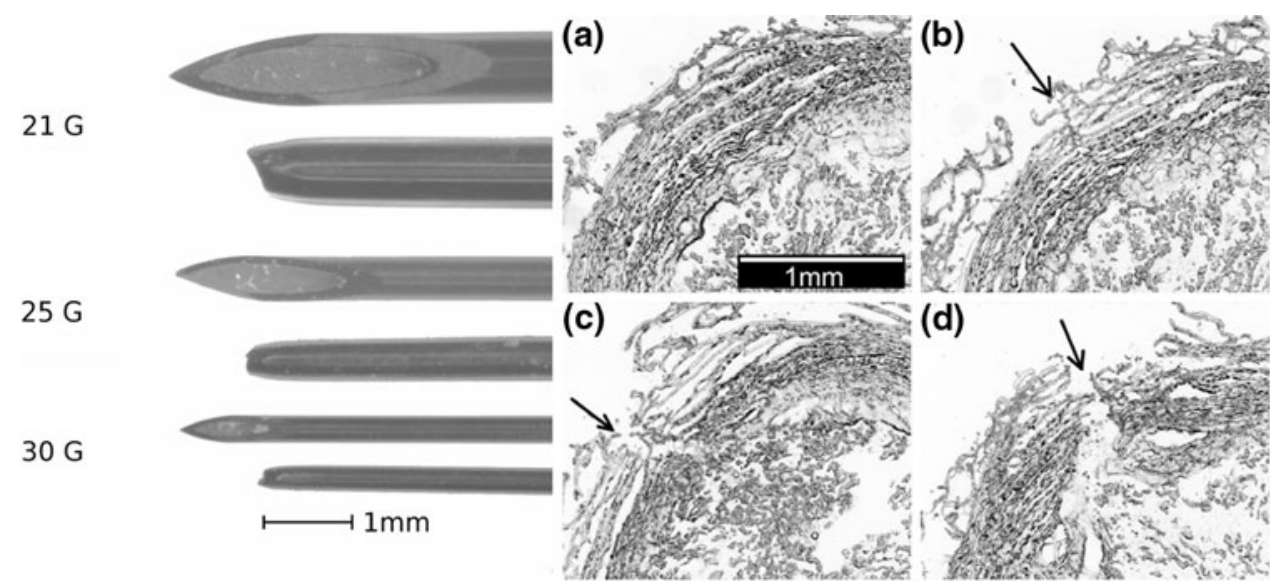

(Fig. 3a) stiffnesses of the discs from force versus displacement measurements. These two parameters independently describe the elastic $\left(K^{\prime}\right)$ and viscoelastic $\left(K^{\prime \prime}\right)$ behaviors of the specimen.

\section{Torsional testing}

Torsional biomechanical properties were obtained using a rheometer (TA Instruments) fitted with the grips described above and a rotation-controlled protocol (Fig. 2b).
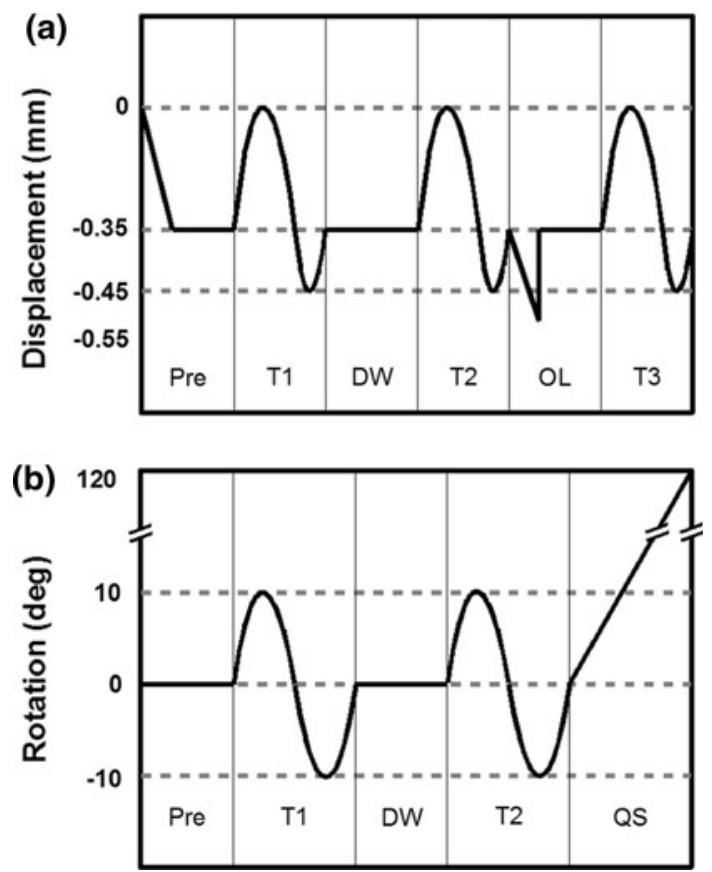

Fig. 2 Schematic representations of loading protocols for compressive test (a), consisting of pre-load (PRE), pre-puncture dynamic test (T1), puncture dwell (DW), post-puncture dynamic test (T2), compressive overload (OL) and post-overload test (T3). Torsional tests (b) consisted of compressive pre-load (PRE), pre-puncture dynamic test (T1), puncture dwell (DW), post-puncture dynamic test (T2) and quasi-static ramp to failure (QS)
Specimens underwent the same axial pre-compression and relaxation procedure as in the compressive test (Pre), followed by a dynamic torsional test consisting of ten cycles of sinusoidal rotation to $\pm 10^{\circ}$ at a frequency of $1 \mathrm{~Hz}$ (T1). This amplitude was chosen to ensure inclusion of behavior both within and surrounding the torsional neutral zone of the disc [23], and was shown to not cause an injury response in vivo [26]. Following testing was a 5-min dwell (DW), during which specimens assigned to the injury groups (control: $n=12,30 \mathrm{G}: n=11,25 \mathrm{G}: n=13$, $21 \mathrm{G}: n=10$ ) were punctured. After puncture, the torsional test was repeated (T2), followed by a ramp from 0 to $120^{\circ}$ at a rate of $1 \% \mathrm{~min}(\mathrm{QS})$. Pilot studies showed that this rotational magnitude was sufficient to cause failure, and that the rate was slow enough to be considered as quasistatic.

Torsional mechanical data were analyzed using two custom-written Matlab codes (Mathworks). The first used dynamic torsional test data to establish linear region stiffness under loading $\left(K_{\mathrm{LR}}\right)$, neutral zone length $\left(L_{\mathrm{NZ}}\right)$ and hysteresis area $\left(A_{\mathrm{H}}\right)$ (Fig. $\left.3 \mathrm{~b}\right)$. The neutral zone stiffness was assumed to be zero due to the high nonlinearity of the data. The second code allowed three independent observers to evaluate the failure ramp data. Observers digitized relevant points on the torque-rotation curves (for specimens randomly ordered without information about the damage groups) to evaluate quasi-static stiffness prior to failure $\left(K_{\mathrm{QS}}\right)$, torque $\left(\tau_{\mathrm{MF}}\right)$ and rotation $\left(\theta_{\mathrm{MF}}\right)$ at the first microfailure event, and torque $\left(\tau_{\mathrm{U}}\right)$ and rotation $\left(\theta_{\mathrm{U}}\right)$ at ultimate failure, as shown (Fig. 3c). Specimens were also categorized as having no micro-failure events, a single event or multiple events.

\section{Statistics}

Two-way ANOVAs evaluated biomechanical parameters of compressive and torsional tests to determine the effects of the intervention (puncture and overload) as well as 
Fig. 3 Dynamic compressive tests were used to calculate storage $\left(K^{\prime}\right)$ and loss $\left(K^{\prime \prime}\right)$ stiffnesses (a). Dynamic torsion tests (b) yielded linear region stiffness $\left(K_{\mathrm{LR}}\right)$, neutral zone length $\left(L_{\mathrm{NZ}}\right)$ and hysteresis area $\left(A_{\mathrm{H}}\right)$. Quasi-static stiffness $\left(K_{\mathrm{QS}}\right)$, torque and rotation at micro-failure ( $\tau_{\mathrm{MF}}$ and $\left.\theta_{\mathrm{MF}}\right)$, and torque and rotation at ultimate failure $\left(\tau_{\mathrm{U}}\right.$ and $\left.\theta_{\mathrm{U}}\right)$ were calculated from the ramp to failure (c)

(a)

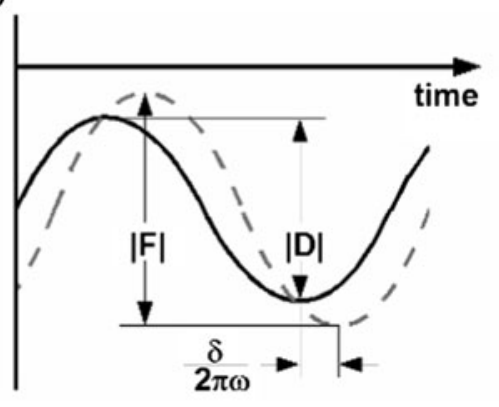

(b)

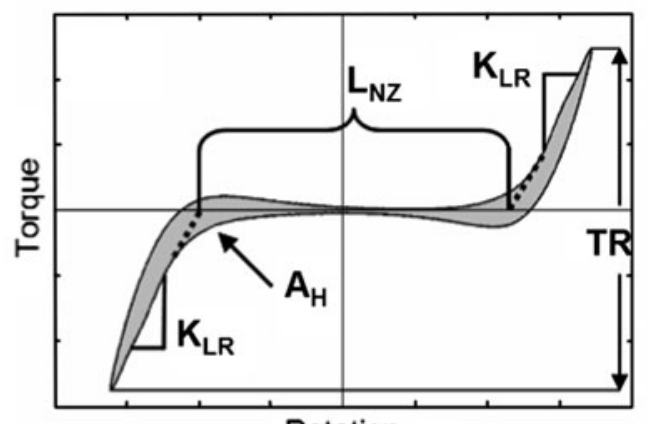

$$
\begin{aligned}
\mathrm{K}^{\prime} & =\frac{|(\mathrm{F})|}{|(\mathrm{D})|} \cos (\delta) \\
\mathrm{K}^{\prime \prime} & =\frac{|(\mathrm{F})|}{|(\mathrm{D})|} \sin (\delta)
\end{aligned}
$$

Rotation

(c)

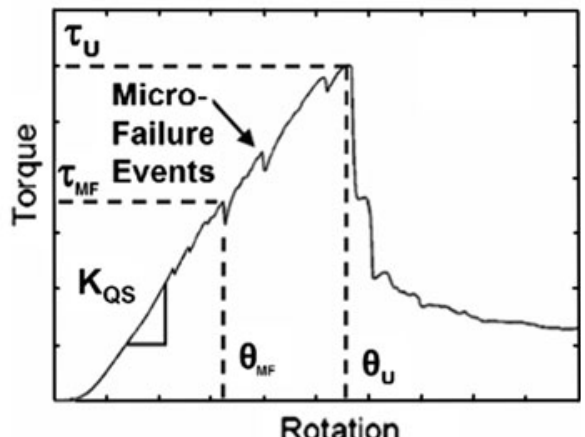

injury group (control $30 \mathrm{G}, 25 \mathrm{G}$ and $21 \mathrm{G}$ ). The effect of needle size on torsional failure ramp parameters was determined using a one-way ANOVA. Post hoc comparisons between punctured and pre-punctured states were performed using paired $t$ tests with $p<0.05$ significant. Comparisons of punctured groups to control involved unpaired $t$ test with significance level adjusted to $p<0.017$ to account for multiple comparisons.

\section{Results}

\section{Compressive test}

Controls showed no difference between pre- and postpuncture stiffness indicating that the first test and subsequent 5-min dwell did not affect compressive mechanics (Table 1). The punctures resulted in a significant $20 \%$ decrease in storage stiffness of all puncture groups with no effect of needle size. Following compressive overload, storage stiffness was reduced to $50 \%$ of pre-punctured, with no difference either between needle sizes or between punctured and unpunctured groups. Loss of stiffness was not affected by either puncture or overload.

\section{Torsional test}

Torsional parameters (Table 2) were affected by the 5 -min dwell, and comparisons were thus made between the injured and control. Punctures resulted in downward trends in linear region stiffness (25 and $21 \mathrm{G}$ significant) and hysteresis area (21 G significant), but no significant changes in the neutral zone length. Needle puncture resulted in no significant changes in the torque or rotation of the first observed micro-failure prior to ultimate failure, but a progressively increasing incidence of micro-failure events (Fig. 4). As much as $77 \%$ of control specimens experienced no micro-failure prior to ultimate failure, compared with $15 \%$ of $21 \mathrm{G}$ specimens. Conversely, fewer than $8 \%$ of control specimens experienced multiple micro-failure events compared with $64 \%$ of $21 \mathrm{G}$ specimens. Significant trends were not observed for other failure parameters. Mean values for all specimens $\pm \mathrm{SD}$ are as follows; $K_{\mathrm{QS}}$ : $2.5 \pm 1.8 \mathrm{Nmm} /{ }^{\circ}, \theta_{\mathrm{MF}}: 38 \pm 14^{\circ}, \tau_{\mathrm{MF}}: 29 \pm 15 \mathrm{Nmm}$, $\theta_{\mathrm{U}}: 59 \pm 10^{\circ}, \tau_{\mathrm{U}}: 48 \pm 14 \mathrm{Nmm}$.

\section{Discussion}

The present comparison of compressive and torsional consequences of needle puncture provides the most comprehensive picture of AF injury in small animal IVDs currently available. AF puncture is generally associated with potential loss of nuclear fluid, which would reduce storage (i.e., elastic) stiffness in compression. The most notable finding of this study, however, was the consistent $20 \%$ reduction in storage stiffness with puncture of all needle sizes, indicating that needle puncture alone had an 
Table 1 Mean dynamic compressive parameters (storage stiffness $K^{\prime}$ and loss stiffness $K^{\prime \prime}$ ) \pm SEM

\begin{tabular}{|c|c|c|c|c|c|c|}
\hline \multirow[t]{2}{*}{ Needle size } & \multicolumn{2}{|l|}{ Pre-puncture } & \multicolumn{2}{|c|}{ Post-puncture } & \multicolumn{2}{|l|}{ Overload } \\
\hline & $K^{\prime}(\mathrm{N} / \mathrm{mm})$ & $K^{\prime \prime}(\mathrm{N} / \mathrm{mm})$ & $K^{\prime}(\mathrm{N} / \mathrm{mm})$ & $K^{\prime \prime}(\mathrm{N} / \mathrm{mm})$ & $K^{\prime}(\mathrm{N} / \mathrm{mm})$ & $K^{\prime \prime}(\mathrm{N} / \mathrm{mm})$ \\
\hline Control & $21 \pm 2.2$ & $0.74 \pm 0.10$ & $20 \pm 2.1$ & $0.72 \pm 0.14$ & $11 \pm 1.0$ & $0.69 \pm 0.11$ \\
\hline $30 \mathrm{G}$ & $16 \pm 1.9$ & $0.52 \pm 0.06$ & $14 \pm 1.9$ & $0.42 \pm 0.08$ & $\mathbf{8} \pm \mathbf{1 . 0}$ & $0.42 \pm 0.07$ \\
\hline $25 \mathrm{G}$ & $19 \pm 2.4$ & $0.59 \pm 0.10$ & $15 \pm 1.8$ & $0.37 \pm 0.08$ & $10 \pm 1.2$ & $0.50 \pm 0.11$ \\
\hline $21 \mathrm{G}$ & $15 \pm 2.2$ & $0.50 \pm 0.07$ & $12 \pm 2.5$ & $0.57 \pm 0.11$ & $7 \pm \mathbf{1 . 3}$ & $0.46 \pm 0.09$ \\
\hline
\end{tabular}

Bold numbers indicate significant $(p<0.05)$ difference between pre- and post-puncture or between post-puncture and overload groups

Table 2 Mean dynamic torsion parameters (linear region stiffness $K_{\mathrm{LR}}$, neutral zone length $L_{\mathrm{NZ}}$, and hysteresis area $A_{\mathrm{H}}$ ) $\pm \mathrm{SEM}$

\begin{tabular}{|c|c|c|c|c|c|c|}
\hline \multirow[t]{2}{*}{ Needle size } & \multicolumn{3}{|l|}{ Pre-puncture } & \multicolumn{3}{|l|}{ Post-puncture } \\
\hline & $K_{\mathrm{LR}}\left(\mathrm{Nmm} /{ }^{\circ}\right)$ & $L_{\mathrm{NZ}}\left(^{\circ}\right)$ & $A_{\mathrm{H}}\left(\mathrm{Nmm}^{\circ}\right)$ & $K_{\mathrm{LR}}\left(\mathrm{Nmm} /{ }^{\circ}\right)$ & $L_{\mathrm{NZ}}\left(^{\circ}\right)$ & $A_{\mathrm{H}}\left(\mathrm{Nmm}^{\circ}\right)$ \\
\hline Control & $2.0 \pm 0.10$ & $12 \pm 0.40$ & $8.9 \pm 1.0$ & $2.3 \pm 0.15$ & $10 \pm 0.49$ & $16 \pm 2.0$ \\
\hline $30 \mathrm{G}$ & $1.8 \pm 0.17$ & $12 \pm 0.47$ & $7.7 \pm 1.5$ & $2.0 \pm 0.17$ & $\mathbf{1 0} \pm \mathbf{0 . 4 0}$ & $18 \pm 1.9$ \\
\hline $25 \mathrm{G}$ & $1.9 \pm 0.17$ & $12 \pm 0.58$ & $8.1 \pm 1.1$ & $1.7 \pm 0.14^{+}$ & $11 \pm \mathbf{0 . 5 5}$ & $13 \pm 1.9$ \\
\hline $21 \mathrm{G}$ & $1.7 \pm 0.14$ & $13 \pm 0.72$ & $6.5 \pm 1.1$ & $1.4 \pm 0.12^{+}$ & $12 \pm 0.54$ & $9 \pm 1.4^{+}$ \\
\hline
\end{tabular}

Bold numbers indicate significant difference $(p<0.05)$ between T1 and T2, and ${ }^{+}$indicates significant $(p<0.017)$ difference between punctured and control specimens

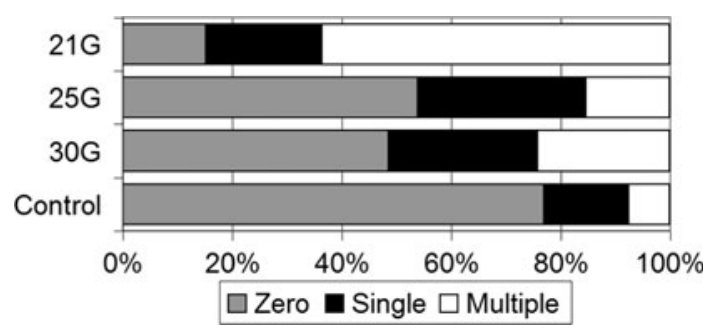

Fig. 4 Rates of micro-failure events by group during quasi-static torsion. Each specimen was classified as experiencing zero, one or multiple micro-failure events

effect that was not associated with injury size. This shows that under slow dynamic compression, the presence of a penetrating anular injury is more important than the injury size. Since the injury effects disappeared following water removal via compressive overload, it was inferred that pressurization of this water provided significant stiffness to the unpunctured disc.

Torsional measurements were very sensitive and showed a significant increase in stiffness following the prepuncture torsional test and 5-min dwell period, which was likely associated with transient disc hydration. Most importantly, an alteration of disc mechanics from puncture depended on needle size and, therefore, extent of anular fiber breakage. Specifically, sensitivity to needle injury was only observed in parameters which depend on fiber tension, such as linear region $\left(K_{\mathrm{LR}}\right)$ and hysteresis area $\left(A_{\mathrm{H}}\right)$. Behavior in the neutral zone, where anular fibers are lax, was not affected by puncture. Increasing needle diameter also increased the likelihood of a disc experiencing a micro-failure event supporting the hypothesis that accumulation of damage at the site of an acute injury is a pathway to disc degeneration. However, needle puncture did not decrease rotations at which the micro-failure first occurred. The lack of a clear trend in torque or rotation at ultimate failure is most likely the result of the high degree of variability in disc behavior following micro-failure combined with inter-specimen variability.

It is important to recognize that ultimate torsional failure occurred at rotations $\left(\sim 60^{\circ}\right)$ far in excess of the expected physiological range for rats. Human lumbar discs have a torsional range of motion limited to only $\sim 2^{\circ}$ by interference between the vertebral facet joints [27] and a neutral zone half-length of approximately $1^{\circ}[4]$. The present study shows a neutral zone half-length of $>5^{\circ}$, suggesting a much greater torsional laxity in rat caudal discs. Interestingly, visual inspection of tested motion segments showed that all torsional failure occurred via separation of the annulus from one of the vertebral endplates (Fig. 5). Neither the mode of failure nor the location of the separation was affected by the presence of a needle puncture. Prior investigation of disc failure under internal pressurization [28] had pointed toward this interface as a weakness, though surprisingly the presence of large annulus injuries in this study did not shift failure to the puncture sites.

The difference in the IVD's response to injury in compressive and torsional loading is most likely the 
Fig. 5 Torsional failure via rim separation in control (left) and $25 \mathrm{G}$ (right) specimens. Black arrows indicate point of failure and white arrow indicates puncture site
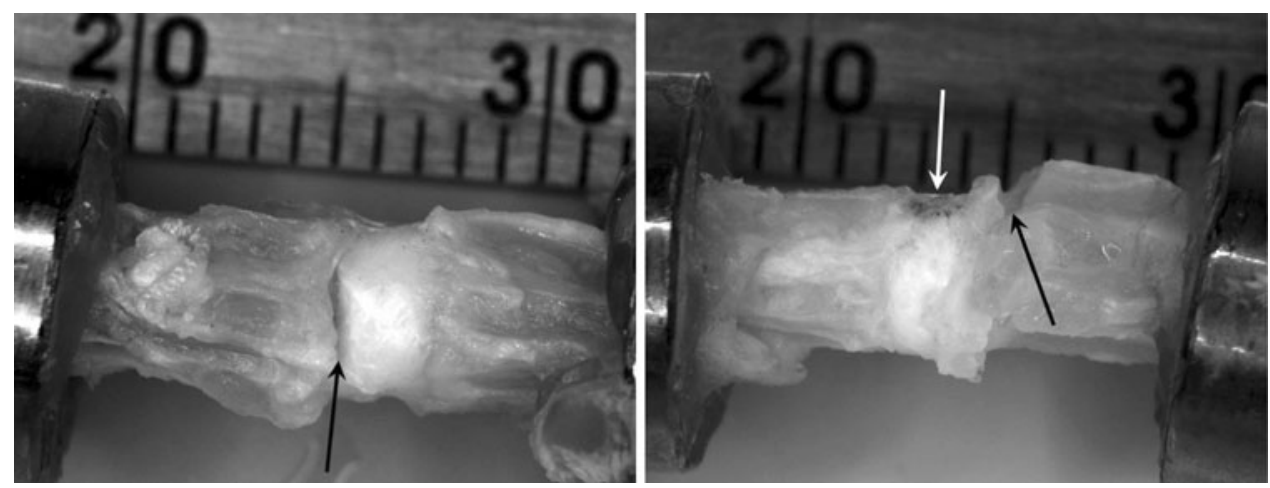

result of altered mechanisms through which these two modes are governed by NP fluid pressurization and AF fiber tension. In human lumbar IVDs, torsion results in significantly higher anular fiber stretch than compression $[29,30]$. AF stiffness is also proportional to the percentage of intact fibers [31, 32], which accounts for the observed decline in linear region stiffness with increasing injury size. The dominance of fluid pressurization on compressive mechanics, along with the lack of needle size effect in compression, indicates that in this mode the presence of a penetrating anular injury is more important than the size of the injury. This finding is consistent with prior measurements showing a loss of disc height resulting only from injuries that fully penetrate the AF [6]. While some migration of nuclear material was observed following puncture (Fig. 1b), the amount of tissue displaced correlated with needle size, while the resulting loss of compressive stiffness did not. A bulk extrusion of NP material was not observed following compression, implying that fluid flow through the tissue was preferential to displacement of the solid matrix. This effect is likely the result of the high (83.5\%) water content of the rat caudal NP [24]. These findings suggests that at the rates of compressive loading used in this study, a needle injury opens up an additional fluid transport pathway, which offers effectively zero resistance to flow.

The highly gelatinous NP of rat caudal IVDs may limit translation of these findings to human IVDs, which contain a more fibrous NP tissue. The small size of rat caudal IVDs also constrains choices of needle size relative to disc height. Needles smaller than 40\% [19] of disc height, a size lying between our 25 and $30 \mathrm{G}$ needles for rats, were shown to not affect disc compressive mechanics. The significant effects of $30 \mathrm{G}$ puncture in this study, therefore, likely resulted from modification of the hypodermic needles as well as differences in loading protocols. Despite the difficulty with translating rodent disc biomechanics to the scale of human discs, these measurements still play in important role in basic disc research. The rat tail IVD is an attractive choice for in vivo studies of disc injury, degeneration and potential repair due to low cost and genetic variation along with ease of access. Accurate and sensitive biomechanical measurements are essential to assess the outcomes of these studies.

The present study has interpreted distinct needle puncture injury effects for compression (associated with introduction of a new fluid flow pathway) and torsion (associated with annulus fiber tension), thereby deepening our understanding of the mechanisms of injury and providing a conceptual model that may be helpful in interpreting injuries in small animal models. It is hoped that these findings will help define design criteria for effective $\mathrm{AF}$ repair techniques and inform selection of test protocols to effectively quantify those repairs.

\section{References}

1. Urban JPG, Roberts S (2003) Degeneration of the intervertebral disc. Arthritis Res Ther 5:120-130

2. Iatridis JC, Michalek AJ, Purmessur D, Korecki CL (2009) Localized intervertebral disc injury leads to organ level changes in structure, cellularity, and biosynthesis. Cell Mol Bioeng 2:437-447

3. Pollintine P, van Tunen MS, Luo J, Brown MD, Dolan P, Adams MA (1976) Time-dependent compressive deformation of the ageing spine: relevance to spinal stenosis. Spine 35:386-394

4. Costi JJ, Stokes IA, Gardner-Morse MG, Iatridis JC (2008) Frequency-dependent behavior of the intervertebral disc in response to each of six degree of freedom dynamic loading: solid phase and fluid phase contributions. Spine 33:1731-1738

5. Carragee EJ, Don AS, Hurwitz EL, Cuellar JM, Carrino J, Herzog R (2009) Does discography cause accelerated progression of degeneration changes in the lumbar disc: a ten-year matched cohort study. Spine 34:2338-2345

6. Aoki Y, Akeda K, An H, Muehleman C, Takahashi K, Moriya H, Masuda K (2006) Nerve fiber ingrowth into scar tissue formed following nucleus pulposus extrusion in the rabbit anular-puncture disc degeneration model: effects of depth of puncture. Spine 31:E774-E780

7. Kim KS, Yoon ST, Li J, Park JS, Hutton WC (2005) Disc degeneration in the rabbit: a biochemical and radiological comparison between four disc injury models. Spine 30:33-37 
8. Korecki CL, Costi JJ, Iatridis JC (2008) Needle puncture injury affects intervertebral disc mechanics and biology in an organ culture model. Spine 33:235-241

9. Miyamoto K, Masuda K, Kim JG, Inoue N, Akeda K, Andersson GB, An HS (2006) Intradiscal injections of osteogenic protein-1 restore the viscoelastic properties of degenerated intervertebral discs. Spine J 6:692-703

10. Sobajima S, Kim JS, Gilbertson LG, Kang JD (2004) Gene therapy for degenerative disc disease. Gene Ther 11:390-401

11. Sobajima S, Kompel JF, Kim JS, Wallach CJ, Robertson DD, Vogt MT, Kang JD, Gilbertson LG (2005) A slowly progressive and reproducible animal model of intervertebral disc degeneration characterized by MRI, X-ray, and histology. Spine 30:15-24

12. Hsieh AH, Hwang D, Ryan DA, Freeman AK, Kim H (2009) Degenerative anular changes induced by puncture are associated with insufficiency of disc biomechanical function. Spine 34:9981005

13. Fazzalari NL, Costi JJ, Hearn TC, Fraser RD, Vernon-Roberts B, Hutchinson J, Manthey BA, Parkinson IH, Sinclair C (2001) Mechanical and pathologic consequences of induced concentric anular tears in an ovine model. Spine 26:2575-2581

14. Holm S, Ekstrom L, Kaigle Holm A, Hansson T (2007) Intradiscal pressure in the degenerated porcine intervertebral disc. Vet Comp Orthop Traumatol 20:29-33

15. Kaigle A, Ekstrom L, Holm S, Rostedt M, Hansson T (1998) In vivo dynamic stiffness of the porcine lumbar spine exposed to cyclic loading: influence of load and degeneration. J Spinal Disord 11:65-70

16. Keller TS, Holm SH, Hansson TH, Spengler DM (1990) 1990 Volvo Award in experimental studies. The dependence of intervertebral disc mechanical properties on physiologic conditions. Spine 15:751-761

17. Thompson RE, Pearcy MJ, Barker TM (2004) The mechanical effects of intervertebral disc lesions. Clin Biomech (Bristol, Avon) 19:448-455

18. Thompson RE, Pearcy MJ, Downing KJ, Manthey BA, Parkinson IH, Fazzalari NL (2000) Disc lesions and the mechanics of the intervertebral joint complex. Spine 25:3026-3035

19. Elliott DM, Yerramalli CS, Beckstein JC, Boxberger JI, Johannessen W, Vresilovic EJ (2008) The effect of relative needle diameter in puncture and sham injection animal models of degeneration. Spine 33:588-596
20. Beckstein JC, Sen S, Schaer TP, Vresilovic EJ, Elliott DM (2008) Comparison of animal discs used in disc research to human lumbar disc: axial compression mechanics and glycosaminoglycan content. Spine 33:E166-E173 (Phila Pa 1976)

21. Elliott DM, Sarver JJ (2004) Young investigator award winner: validation of the mouse and rat disc as mechanical models of the human lumbar disc. Spine 29:713-722 (Phila Pa 1976)

22. O'Connell GD, Vresilovic EJ, Elliott DM (2007) Comparison of animals used in disc research to human lumbar disc geometry. Spine 32:328-333 (Phila Pa 1976)

23. Espinoza Orias AA, Malhotra NR, Elliott DM (2008) Rat disc torsional mechanics: effect of lumbar and caudal levels and axial compression load. Spine J 9:204-209

24. Masuoka K, Michalek AJ, MacLean JJ, Stokes IA, Iatridis JC (2007) Different effects of static versus cyclic compressive loading on rat intervertebral disc height and water loss in vitro. Spine 32:1974-1979

25. MacLean JJ, Lee CR, Alini M, Iatridis JC (2005) The effects of short-term load duration on anabolic and catabolic gene expression in the rat tail intervertebral disc. J Orthop Res 23:1120-1127

26. Barbir A, Godburn KE, Michalek AJ, Lai A, Monsey RD, Iatridis J (2009) In vivo response of intervertebral discs to static and dynamic torsion. Orthopaedic Research Society, Las Vegas, NV

27. Pearcy M, Portek I, Shepherd J (1984) Three-dimensional X-ray analysis of normal movement in the lumbar spine. Spine 9:294297 (Phila Pa 1976)

28. Veres SP, Robertson PA, Broom ND (2008) ISSLS Prize winner: microstructure and mechanical disruption of the lumbar disc annulus part II: how the annulus fails under hydrostatic pressure. Spine 33:2711-2720

29. Stokes I, Greenapple DM (1985) Measurement of surface deformation of soft tissue. J Biomech 18:1-7

30. Stokes IA (1987) Surface strain on human intervertebral discs. J Orthop Res 5:348-355

31. Adams MA, Green TP (1993) Tensile properties of the annulus fibrosus I. The contribution of fibre-matrix interactions to tensile stiffness and strength. Eur Spine J 2:203-208

32. Krismer M, Haid C, Rabl W (1996) The contribution of anulus fibers to torque resistance. Spine 21:2551-2557 